\title{
Orbital Subperiosteal Hematoma in the Newborn Causing Unilateral Proptosis: Ultrasound as First-Line Imaging Modality
}

\author{
Florencia Angkasa, MBBS ${ }^{1}$ Leila Mohammadi, MLIS² Deepa Taranath, MS, DipNB, FRANZCO ${ }^{3}$
} Ajay Taranath, MBBS, MD (Radiology), FRANZCR ${ }^{4,5}$ Marcus Brecht, Dr.med ${ }^{1}$

${ }^{1}$ Department of Perinatal Medicine, Flinders Medical Centre,

Address for correspondence Marcus Brecht, Dr.med, Department of South Australia, Australia

2 Flinders University, South Australia, Australia

${ }^{3}$ Flinders Eye Centre, Flinders Medical Centre, South Australia, Perinatal Medicine, Flinders Medical Centre, Bedford Park, SA 5042 Australia

${ }^{4}$ Institute for Medical Imaging, Flinders Medical Centre, South Australia, Australia

${ }^{5}$ Department of Radiology, Women's and Children's Hospital, North Adelaide, Australia South Australia, Australia (e-mail: marcus.brecht@sa.gov.au).

Am J Perinatol Rep 2017;7:e138-e143.
Abstract
Proptosis in the neonatal period is relatively infrequent and has diverse underlying etiologies. One of the more common causes appears to be orbital subperiosteal hematoma. Early detection, differentiation from other causes, and regular follow-up
Keywords
- newborn
- orbital
- subperiosteal
- hematoma
- proptosis
- ultrasound are essential as loss of vision can occur. We describe two cases of neonatal proptosis caused by orbital subperiosteal hematoma highlighting different diagnostic and management approaches, and provide a summary of previously reported cases. Spontaneous resolution occurs in most cases; however, emergent surgical evacuation is warranted in cases of optic nerve compression. This is the first report to provide orbital ultrasound images of uncomplicated neonatal orbital subperiosteal hematoma. Orbital ultrasound followed by magnetic resonance imaging (MRI) is a valid nonradia- tion approach for assessing neonatal proptosis due to subperiosteal orbital hematoma.

\section{Case Report}

Case 1

A male infant was born at term to a 31-year-old, gravid 3 para 2 mother by precipitous spontaneous vaginal birth. Pregnancy and third trimester fetal ultrasound scan were unremarkable. Apgar scores were 7 ( 1 minute) and 9 ( 5 minutes). Birth weight was $3,815 \mathrm{~g}$ (75th percentile). Facial suffusion was noted immediately after birth and intramuscular vitamin $\mathrm{K}$ was administered shortly after birth.

At 16 hours of age, proptosis of the right eye was noted with associated subconjunctival hemorrhage, right-sided hypotropia, and impaired right lateral gaze (-Fig. 1a). The cornea appeared clear bilaterally, pupils responded to light

received

October 10, 2016

accepted after revision

March 27, 2017
DOI https://doi.org/

10.1055/s-0037-1603953. ISSN 2157-6998. symmetrically, both fundi and the baby's physical examination were unremarkable. A full blood count and coagulation profile were unremarkable (results not shown).

\section{Imaging and Clinical Course}

Orbital ultrasound demonstrated a well-defined hypoechoic, nonvascular $13 \times 9 \mathrm{~mm}$ mass in the superior aspect of right orbit, exerting mass effect and scalloping the retroorbital fat and superior rectus muscle ( $\mathbf{- F i g}$. 1 b, calipers, contralateral eye not shown). A cranial magnetic resonance imaging (MRI) study on day 12 of life demonstrated an ellipsoid lesion measuring $19.8 \times 22.8 \times 11 \mathrm{~mm}$ with elevation of the roof of the right orbit. The lesion was hyperintense on $\mathrm{T} 1$ and showed mixed intensity on T2 sequences (-Fig. 1c). There
Copyright $\odot 2017$ by Thieme Medical Publishers, Inc., 333 Seventh Avenue, New York, NY 10001, USA. Tel: +1(212) 584-4662.
License terms

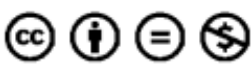



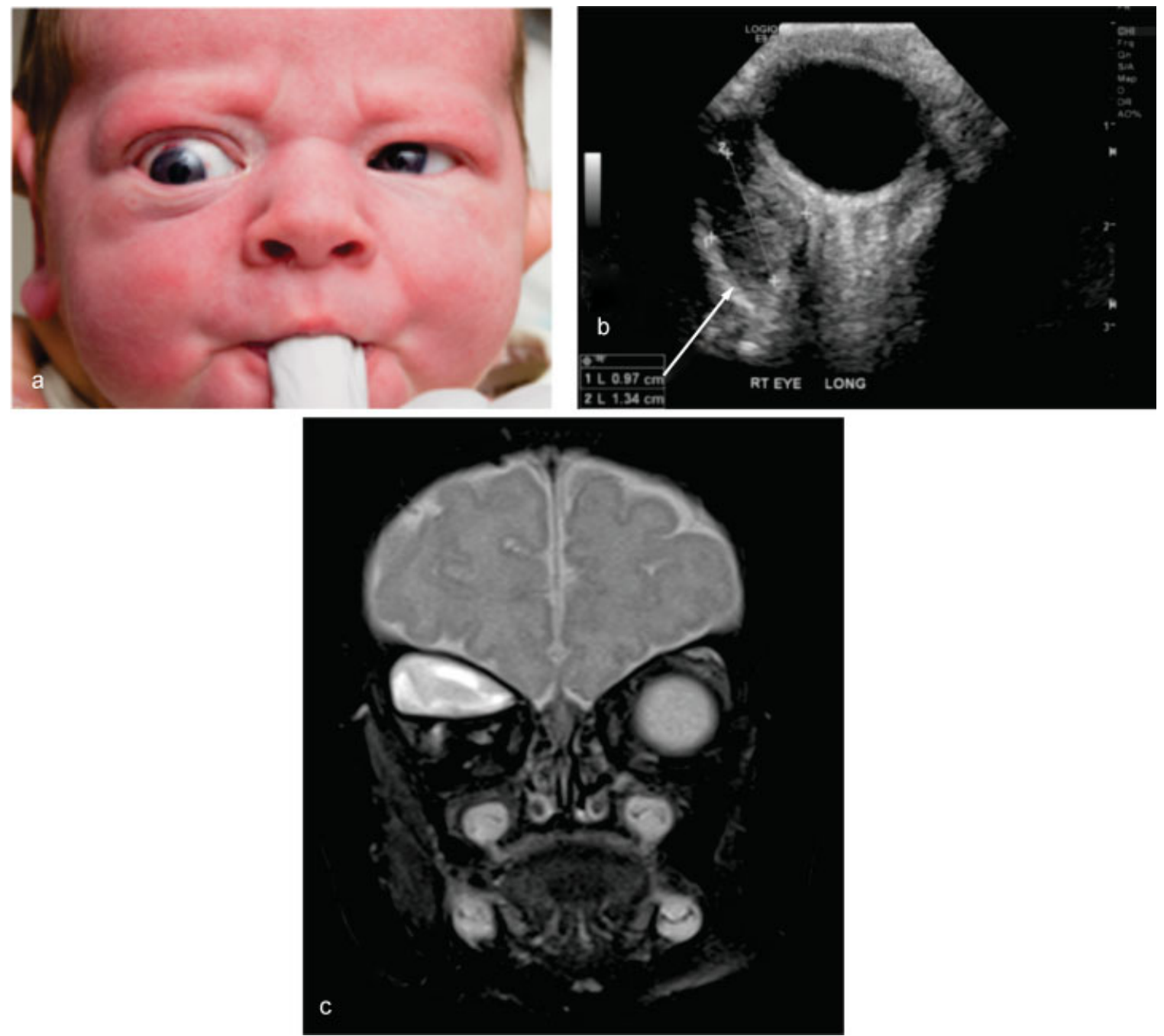

Fig. 1 (a) Proptosis of the right eye with subconjunctival hemorrhage, right-sided hypotropia, and impaired right lateral gaze. (b) Orbital ultrasound (right eye); calipers and arrow indicate retroorbital hematoma. (c) Orbital magnetic resonance imaging (MRI) T2 coronal image showing mixed-intensity, predominantly hyperintense mass.

was no obvious breach involving the bony roof of the orbit to suggest the possibility of a meningocele or a meningoencephalocele. The infant was followed up closely and lubricating eye drops were applied to avoid exposure keratopathy. The proptosis resolved clinically over the course of 10 weeks. The infant had normal visual outcome at the last assessment at 12 months of age.

\section{Case 2}

A male infant was born at term by normal vaginal delivery to a 23-year-old primigravida mother. Pregnancy, antenatal course including fetal third trimester ultrasound, and family history were unremarkable. Birth weight was $3,600 \mathrm{~g}$ (50-75th percentile), length and head circumference were on the 50th percentile, and Apgar scores were 4 ( 1 minute) and 7 (5 minutes). Facial suffusion was noted at birth and prophylactic intramuscular vitamin $\mathrm{K}$ was administered. At about 2 hours after birth, mild swelling of the left upper eyelid was noted; this increased over the following 20 hours with worsening chemosis, proptosis, and subconjunctival hemorrhage
(-Fig. 2a). Empirical treatment with intravenous flucloxacillin, cefotaxime, and acyclovir was commenced. At 55 hours of life, an afferent pupillary deficit was noted. Full blood count and coagulation profile including individual factor analysis were unremarkable (results not shown).

\section{Imaging and Clinical Course}

Emergency computed tomography (CT) imaging demonstrated a nonenhancing ovoid mass measuring $2.2 \times 1 \times 2 \mathrm{~cm}$ in the superior left orbit with mass effect on the left globe and left optic nerve ( - Fig. 2 b). No fracture was observed. On orbital MRI, a uniform mass without enhancement, calcification, or cysts was noted ( $\mathbf{F i g}$. 2c). The left optic nerve was displaced inferiorly ( - Fig. 2d).

Emergency orbital decompression at 65 hours of age via left upper lid sub-brow incision was uncomplicated. Followup at 3, 6, and 12 months of age demonstrated normal fundi and visual capacity. Repeat orbital MRI at 4 months showed normal anatomy and normal-caliber optic nerves (images not shown). 


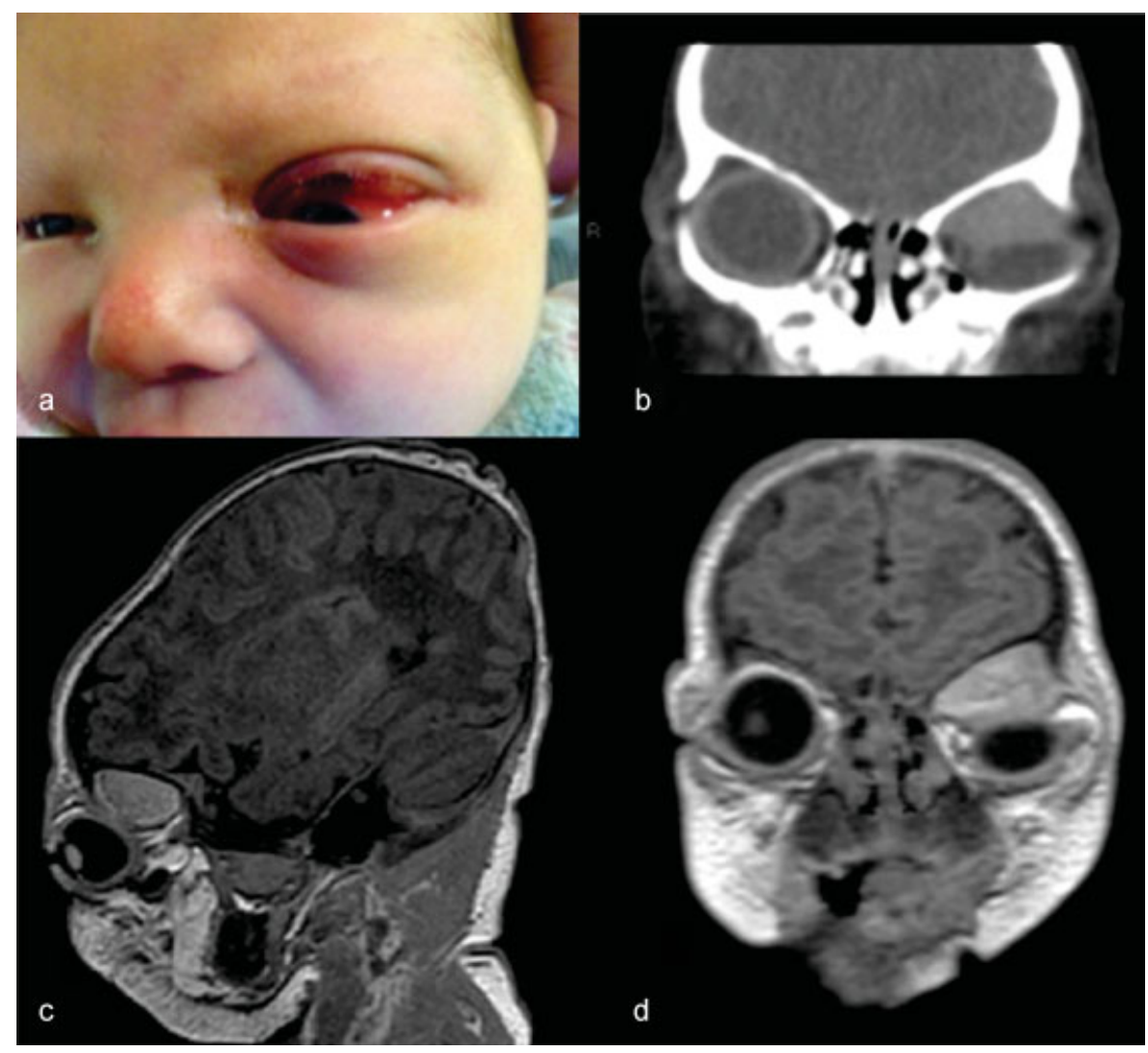

Fig. 2 (a) Left-sided proptosis with subconjunctival hemorrhage. (b) CT demonstrating ellipsoid mass in the left superior orbit. (c) Orbital magnetic resonance imaging (MRI, T1 sequence, sagittal image) showing compression of the left optic nerve by the hyperintense ovoid mass. (d) Orbital magnetic resonance imaging (MRI, T1 sequence, coronal image) showing the hyperintense ovoid mass in the left superior orbit.

\section{Discussion}

Proptosis at or shortly after birth appears to be relatively uncommon but may potentially be underreported. ${ }^{1}$ Differential diagnoses include congenital tumors (dermoid cyst, teratoma, retinoblastoma, rhabdomyosarcoma, neuroblastoma, neurofibroma, myofibroma), vascular malformations, lymphangiomas, cysts, encephalocele/meningomyelocele, bony abnormalities, abscesses, and thyrotoxicosis. ${ }^{1}$

Subperiosteal orbital hemorrhage is a recognized but rarely reported entity. A systematic literature review yielded seven cases with available imaging along with relevant details, which are summarized in - Table $\mathbf{1}^{2-8}$ (see Supplementary Material for search strategy [online only]). The majority of these cases were unilateral; bilateral involvement was described in two cases. ${ }^{6,7}$

The mechanism of neonatal subperiosteal orbital hemorrhage is not entirely clear. A history of traumatic delivery has often been quoted. Among reported cases, only one case was associated with forceps and one case with vacuum-assisted delivery; ${ }^{2,5}$ two cases occurred in the context of shoulder dystocia ( - Table 1) ${ }^{4,8}$ Extrapolating from adult case series, the presumed pathogenesis could be a sudden rise in intraabdominal and intrathoracic venous pressure, and transmission of this pressure into the valveless orbital veins during birth. ${ }^{4}$ Another contributing factor could be the vacuum created upon disimpaction of head during birth. ${ }^{4,9}$ This has been postulated in a newborn after failed rotational forceps delivery, disimpaction of the head, and subsequent delivery by cesarean section. ${ }^{9}$ In this case, marked proptosis was noted; noncontrast CT, however, excluded intraorbital hemorrhage. ${ }^{9}$ In our cases, both infants had facial suffusion, which would lend support to a rise in facial venous pressure. The loose attachment of the periosteum to the bone in the superolateral aspect of the orbit in infants may explain the characteristic location in all reported cases, including the two cases presented here. ${ }^{2-8,10}$ In one previous case, a subperiosteal hematoma with periosteal defect leading to leaking of blood into the orbit was found at the time of surgical exploration. ${ }^{3}$

Coagulopathy does not seem to be common as evident from the normal results of coagulation screens obtained in the reported cases. ${ }^{2-8}$ Administration of vitamin $\mathrm{K}$ at birth needs to be ascertained in all cases. In one infant, preexisting coagulopathy and thrombocytopenia were aggravated by anticoagulation for extracorporeal membrane oxygenation (ECMO) for cardiorespiratory failure; ${ }^{5}$ this infant developed proptosis secondary to subperiosteal orbital hematoma, which was confirmed by contrast-enhanced MRI and which resolved spontaneously. ${ }^{5}$ Proptosis and hyphema were the only initial manifestations in a premature, growth-restricted infant subsequently diagnosed with neonatal alloimmune thrombocytopenia (NAIT) without any other clinical signs suggestive of thrombocytopenia such as petechiae. Details 


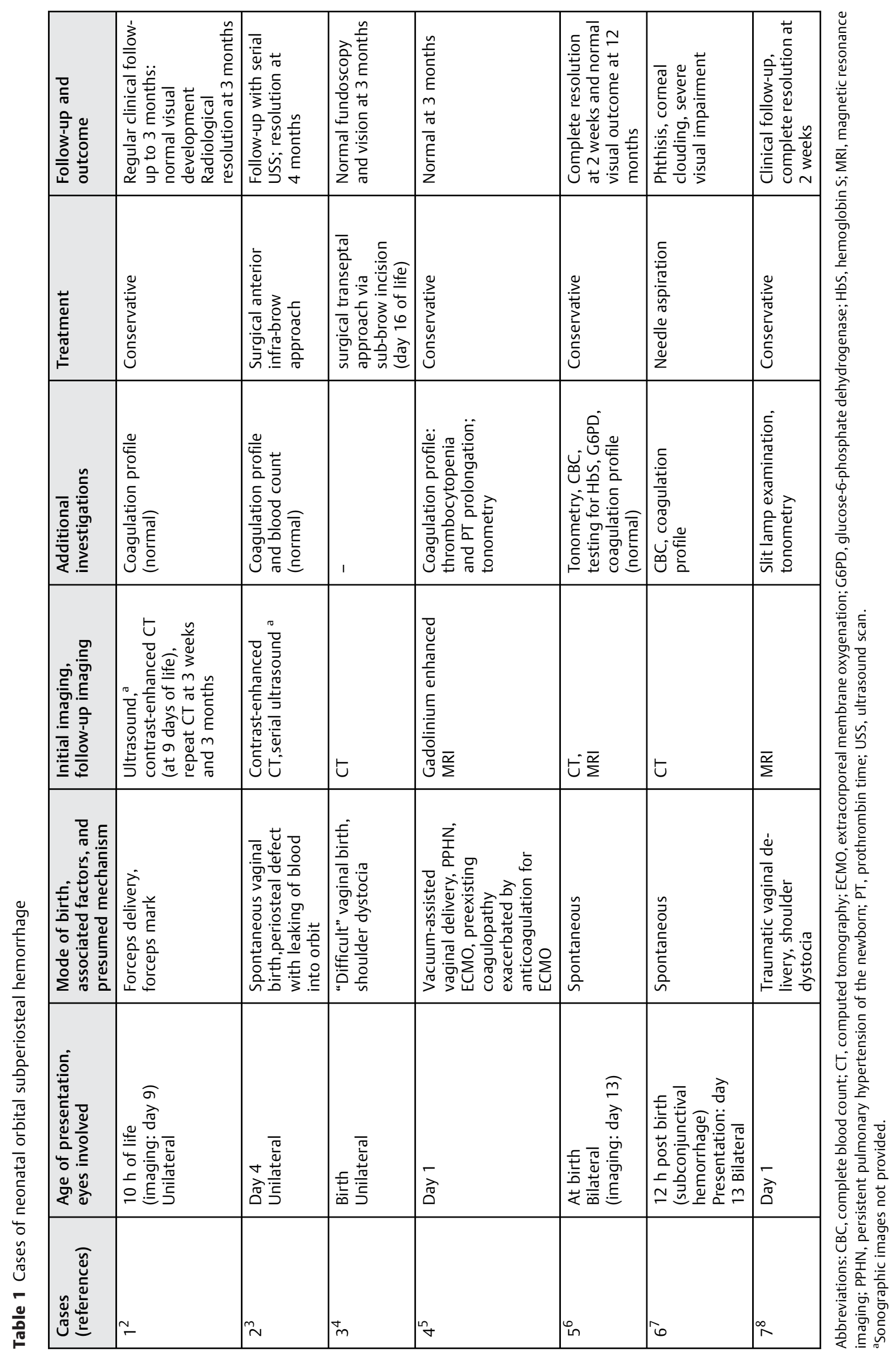


on orbital imaging for this case were not provided. ${ }^{11}$ Another newborn presenting with proptosis from birth secondary to intraorbital hemorrhage was subsequently diagnosed with factor VII deficiency; of note, there was no family history of bleeding diathesis. ${ }^{12}$ No imaging details were provided in this case as to the exact anatomical location of the hemorrhage. ${ }^{12}$ Other uncommon causes of proptosis should also be considered. In an older child, "atypical" frontal cephalhematoma with intraorbital extension has been reported following minor head trauma. ${ }^{13}$ Extensive subgaleal hematoma with extension into the orbit has recently been described in a 10-year-old girl with factor VII deficiency after head trauma. ${ }^{14}$ To our knowledge, both entities have not been reported in the neonatal age group. In view of the possibility of NAIT and substantial intravascular blood loss with both subgaleal and cephalhematoma, a full blood count should be included in the initial workup in any newborn presenting with proptosis and repeated depending on the progress.

Diagnosis of subperiosteal orbital hemorrhage can be confirmed by a variety of imaging modalities, including ultrasound, orbital CT, and MRI. Assessment of ocular pressure with tonometry has been reported in three cases. ${ }^{5,6,8}$ of the seven cases reviewed, six, including the second infant in our series, had undergone an orbital CT at some stage; in two cases, this was the only imaging modality. ${ }^{2-8}$ Orbital MRI was used in three cases, in two of which, it was the only imaging modality. ${ }^{5,8}$ The advantage of MRI in this context is the lack of radiation exposure, ability to reliably exclude an orbital encephalocele, and to differentiate blood at different stages of degradation. ${ }^{6,8,15,16}$ Orbital ultrasound has been employed in two cases as initial imaging modality in one and for follow-up in the second case, but no images have been provided. ${ }^{2,3}$ Reported ultrasonographic features include a well-demarcated homogenous retroorbital mass with low to medium reflectivity and sound attenuation and absence of calcification. ${ }^{2,3}$ In the case reported by Kurlemann et al, orbital ultrasound on day 9 of life demonstrated a homogenous, clearly demarcated hypoechoic/cystic lesion consistent with a hematoma in resorption. ${ }^{2}$ In summary, these findings are in keeping with the imaging features in case 1 presented here. It seems appropriate to obtain an orbital ultrasound initially; if, however, suspicion of orbital fracture is high, $\mathrm{CT}$ is recommended due to its widespread availability and ability to exclude bony abnormalities. ${ }^{16}$ An additional clue for retroorbital hemorrhage as the underlying cause for proptosis in the newborn is a normal third trimester ultrasound, possibly evidence of periorbital trauma with instrumental delivery and conjunctival congestion or frank subconjunctival hemorrhage. ${ }^{2-8}$

The management of retroorbital hemorrhage is largely conservative with an aim to prevent exposure keratitis. However, evacuation of the hematoma has been performed to reduce pressure on the optic nerve or because of diagnostic uncertainty in three of the seven reported cases and in the second case in our series. ${ }^{3,4,7}$ Although duration of follow-up was short in some cases and varied from 2 weeks to 12 months, the majority of neonatal cases had a normal visual outcome including the current two cases. Of the reported cases, one infant suffered from severe visual impairment due to phthisis despite needle aspiration, possibly because of significant exposure keratitis. ${ }^{7}$

In summary, neonatal orbital subperiosteal hematoma is relatively rare but should be considered in any newborn presenting with proptosis. This case series and literature review summarizes the spectrum of severity seen with orbital subperiosteal hematoma in the newborn and highlights the need for early decompression in cases with optic nerve dysfunction. This is also the first report to provide orbital ultrasound images of neonatal orbital subperiosteal hematoma and supports a noninvasive, "no radiation" approach as first-line imaging in mild cases without afferent pupillary deficit. It also highlights the advantages of combining ultrasound and later MRI to demonstrate resolution of the hematoma alongside close clinical review to avoid unnecessary surgical intervention in milder cases.

\section{Note}

No funding was received for this article.

\section{Conflict of Interests}

None of the authors has any conflict of interests to declare. Written informed consent was obtained from the families of the infants prior to submission.

\section{References}

1 Erickson BP, Tse DT. Management of neonatal proptosis: a systematic review. Surv Ophthalmol 2014;59(04):378-392

2 Kurlemann G, Schlüter A, Dominick HC. Subperiostales Hämatom der Orbita nach Zangenentbindung-Kasuistik. (in German). Geburtshilfe Frauenheilkd 1987;47(01):61-62

3 Munoz M, Weatherhead R. Orbital hemorrhage in a newborn. Am J Ophthalmol 1989;108(02):206-208

4 Skull SA, Sullivan TJ. Subperiosteal orbital haematoma presenting as proptosis at birth. J Paediatr Child Health 1997;33(03): 256-258

5 Bart DJ, Lueder GT. Orbital hemorrhage following extracorporeal membrane oxygenation in a newborn. J Pediatr Ophthalmol Strabismus 1997;34(01):65-67

6 Ezzadin EM, Liu D, Al-Rashed W, Jacquemin C. Bilateral orbital hemorrhage in a newborn. Am J Ophthalmol 2000;129(04): 531-533

7 Kaur A, Misra S. Bilateral subperiosteal haemorrhage in a newborn. Br J Ophthalmol 2013;97(06):793

8 Javaid FZ, Bates A, Conry B, Gowda R. Neonatal orbital subperiosteal haematoma following shoulder dystocia. Arch Dis Child Fetal Neonatal Ed 2016;101(05):F479

9 Preece JM, Cornette L, El-Hindy N. Simple management of isolated proptosis at birth. Arch Dis Child Fetal Neonatal Ed 2005;90(03): F234

10 Tonami H, Kuginuki Y, Okimura T, Yamamoto I, Kawakami S, Sasaki K. MRI of subperiosteal hematoma of the orbit. J Comput Assist Tomogr 1994;18(04):549-551

11 Jerónimo M, Azenha C, Mesquita J, Pereira DF. A rare manifestation of neonatal alloimmune thrombocytopaenia. BMJ Case Rep 2014;2014:pii:bcr2014204393

12 Punnen A, Loganathan SK, Veetiyil GI, Scott JX, Kumar S. Neonate with orbital bleed. Pediatr Blood Cancer 2015;62(03):548 
13 Lee KS, Bae WK, Lee HS, Bae HG, Yun IG. Proptosis from a cephalhematoma in a twelve-year-old girl-a case report. J Korean Med Sci 1991;6(03):251-254

14 Jenkins TL, Zheng CX, Murchison AP, Bilyk JR. Orbital compartment syndrome following post-traumatic subgaleal hematoma. Ophthal Plast Reconstr Surg 2017;33(02):e33-e36
15 Knopp U, Knopp A, Stellmacher F, et al. A non-midline sphenoorbital encephalocele in a newborn. Cent Eur Neurosurg 2009;70 (01):43-47

16 Burns NS, Iyer RS, Robinson AJ, Chapman T. Diagnostic imaging of fetal and pediatric orbital abnormalities. AJR Am J Roentgenol 2013;201(06):W797-W808 